\title{
Research on Liquid Water Distribution in PEMFC Cathode Porous Media
}

\author{
Hongjian Liu ${ }^{l}$, Guodong Zhang ${ }^{1}$, Zeting $\mathrm{Yu}^{1}$, Da Li ${ }^{1}$, Guihua Wang ${ }^{1}$, Congkang Wang ${ }^{2}$, \\ Shuzhan Bai ${ }^{1, *}$, Guoxiang $\mathrm{Li}^{1, * * *}$ \\ ${ }^{1}$ School of Energy and Power Engineering, Shandong University, Jinan 250061, China; \\ ${ }^{2}$ Suzhou Foresight Energy Technology Co., Ltd., Suzhou 215300, China) \\ "E-mail: baishuzhan@sdu.edu.cn, liguox@ @sdu.edu.cn
}

doi: $10.20964 / 2020.07 .27$

Received: 2 March 2020 / Accepted: 22 April 2020 / Published: 10 June 2020

\begin{abstract}
A mass transfer model that modifies the Bruggeman equation for oxygen diffusion in the gas diffusion layer (GLD) has been proposed and validated by experiments. The experimental results indicate that the modified model can accurately simulate the actual working conditions of proton exchange membrane fuel cell (PEMFC) during concentration polarization. The differences of numerical simulation results between before and after modification are further compared, and the distribution characteristics of liquid water in porous media are explored. The results reveal that liquid water saturation and oxygen concentration predicted by the traditional Bruggeman equation are both higher, and for different models the smaller the value of $\beta$ (or the absolute value of $\alpha$ ) is, the more the liquid water will be. The liquid water is accumulated and oxygen mass transfer resistance is enhanced due to increased current density. The reaction rate decreases during concentration polarization, and the generation rate of liquid water slows down accordingly. The growth rate of liquid water and the decrease rate of oxygen concentration under rib are larger than those under channel. The liquid water accumulates much more significantly under rib than under channel at a common working voltage of around $0.55 \mathrm{~V}$. As GDL porosity decreases, liquid water under channel increases almost linearly while the change of liquid water saturation under rib exhibits a parabolic trend. On the whole, liquid water is slightly saturated when porosity is greater than 0.65 . Smaller GDL pore size will considerably increase 'flooding' risk, and thinner GDL thickness is beneficial to the removal of liquid water.
\end{abstract}

Keywords: PEMFC; Liquid water distribution; Porous media; Numerical simulation

\section{$\underline{\text { FULL TEXT }}$}

(C) 2020 The Authors. Published by ESG (www.electrochemsci.org). This article is an open access article distributed under the terms and conditions of the Creative Commons Attribution license (http://creativecommons.org/licenses/by/4.0/). 